\title{
DETERMINATION OF NECESSARY AND SUFFICIENT OPERATIONAL CONDITIONS OF THE SLEEVE-TYPE BEARINGS IN AUTONOMOUS POWER PLANTS OF ORMAT TYPE UNDER CONDITIONS OF SIBERIA AND THE FAR EAST
}

\author{
Olga V. Vysokomornaya ${ }^{1,{ }^{*}, \text { Vladimir S. Vysokomorny }}{ }^{1}$, Alexander S. Beck ${ }^{1}$, Andrey G. \\ Kosintsev $^{1}$, and Vladimir F. Panin \\ ${ }^{1}$ National Research Tomsk Polytechnic University, 634050 Tomsk, Russia
}

\begin{abstract}
The analysis of integral reliability characteristics for autonomous energy sources in gas pipelines of Russia is carried out. The reasons of the most frequent failures are determined. Recommendations for increase of integral reliability characteristics for autonomous energy sources are formulated.
\end{abstract}

\section{Introduction}

No-break supply of manufacturing plant with energy resources is an important factor of stability of production processes and their effective functioning [4]. In most cases, power supply of industrial enterprises is carried out by a centralized power supply networks. However, because of the emergence of new consumers of energy resources in areas geographically remote from centralized power grids, it is necessary to find other costeffective energy sources [2, 3]. Often the problem of energy supply of remote manufacturing plants is solved through the use of stand-alone power plants. In particular, for the operation of auxiliary equipment of industrial enterprises transporting hydrocarbon raw materials (including remotely controlled items, radio relay stations, etc.) in Eastern Siberia and the Far East of Russia the thermal power stations of small capacity (Mini-TPS) with closed thermodynamic steam cycle (fig. 1) are successfully used.

\section{Target of research}

Mini-CHP Plant fully autonomous and automate, uses natural gas as fuel, comply with the requirements between periods of planned servicing and repair upon condition running in compliance with demands of factory of origin. Frequently environmental withstand in Eastern Siberia and the Far East of Russia characterized by high differences temperature

\footnotetext{
*Corresponding author: vysokomornyy@yandex.ru
} 
fall of outward. This application environment may be reasons of appearance of not restricted operating regime in work of mini-CPH-plant connected with temperature disparity of power fluid with factory of origin demands. Above-noted disparity is the cause of failure mini-CPH-plant due to high temperature of leaving aerial condenser organic fluid $[1,6]$.

Due to the fact that the constructive features of observe autonomous power facilities (fig. 1) was using organic fluid such as oiling matter for turbine shaft bearing, recycled after cooling of the aerial condenser, providing the required parameters of the power fluid is a determining factor for ensuring the smooth operation of the power plant.

When temperature of power fluid for bearing lubrication more, than recommended value of factory [5] take place risk of technical deficiency appearance in work of turbinegenerator of mini-CHP plant.

For prevention emergency situation in power plants work it's necessary to determine causes and develop recommendations for decrease failure in work of autonomous miniCHP plant due to mismatch application environment requirements of the factory.

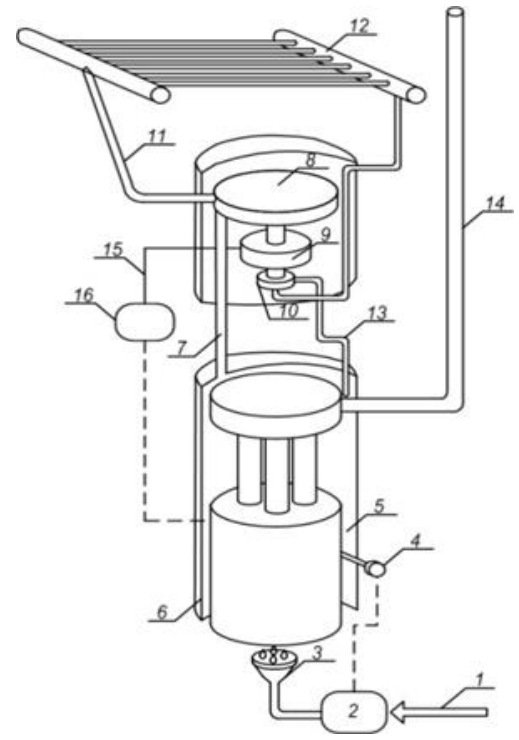

Fig. 1. mini-CHP plant scheme with closed steam cycle: 1 - fossil fuel supply line; 2 - control panel of fuel supply; 3 - fuel burner; 4 - thermostat; 5 - organic liquid; 6 - steam generating unit; 7 - steam line; 8 - turbine; 9 - alternator; 10 - pump; 11 - steam supply tube to condenser; 12 - condenser; 13 - tube of condensate output; 14 - chimney; 15 - AC input cables to the rectifier; 16 - electrical switchboard.

Physical and mathematical formulation of solvable problem of heating transfer present in papers $[1,6]$. Vaporizing working agent from turbine 8 pass to aerial condenser 12 , where heat transfer occurs from pipe wall to ambient air. Then coolant condensate goes to steam power boiler for heating and vaporation passing and lubricating sleeve-type bearings of turbine-generator. Mathematical model, which describe condensing process of working agent includes energy equation for vapor of working agent, heat-transfer equation for film of condensate and pipe wall, diffusion equation, moving, equation of through flow and constitutive equation of working fluid vapor. Nonstationary differential equation system with appropriate initial and boundary conditions solved by finite difference method with using four-point implicit difference scheme $[1,6]$. 


\section{Statistic of the most typical technical deficiency in mini-CPH- plant work}

Figure 2 shows the relevant statistics of typical technical accidents in the work of 101 miniTPS operating in Eastern Siberia and Russian Far East for the period from 2012 to 2015, and earlier accumulated statistics for the period from 2008 to 2012.

The positive dynamics in reducing the number of technical accidents due the "extremely high temperature in the condenser" is associated with the recommendations presented by Vysokomorny V.S. [1] of increase of the reliability and resource efficiency in mini-TPS, operating in cogeneration mode. Because of this recommendations the number of accidents due the "extremely high temperature in the condenser" decreased by 4.9 times. Analysis of the second most important technical accident related to the "mechanical failure of turbogenerator" leads to the conclusion that the probability of its occurance is $35 \%$ / This statistic proves that the need for analysis of occurrence of this accident is an critical task witch requires a detailed study of the appearance causes.

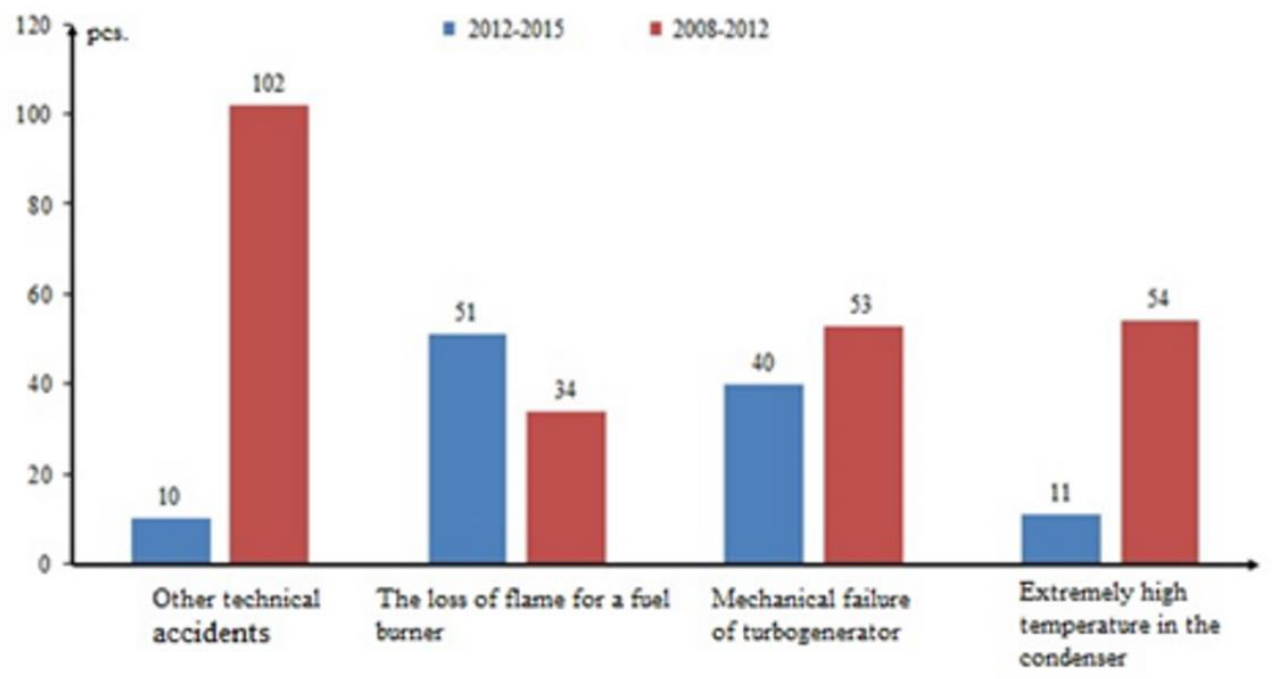

Fig. 2. Statistics of the most typical technical accidents in the work of mini-CHP plant, depending on the year.

\section{Results of numerical simulation}

Figure 3 shows the numerical results of the integral characteristics of the condensation process in the tube, depending on various external air temperature $T_{\text {out }}$ values when the workflow temperature changes entering the tube $T_{\text {output }}$. Numerical simulation of heat and mass transfer processes in the conditions of intensive phase transitions is performed at the following values of thermal characteristics of the interacting substances: $C_{1}=1300(\mathrm{~J} / \mathrm{kg} \cdot \mathrm{K})$, $\rho_{1}=5.1 \mathrm{~kg} / \mathrm{m}^{3}, \lambda_{1}=0.1 \mathrm{~W} /(\mathrm{m} \cdot \mathrm{K}) ; C_{2}=1300(\mathrm{~J} / \mathrm{kg} \cdot \mathrm{K}), \rho_{2}=1300 \mathrm{~kg} / \mathrm{m}^{3}, \lambda_{2}=0.12 \mathrm{~W} /(\mathrm{m} \cdot \mathrm{K}) ;$ $C_{3}=460(\mathrm{~J} / \mathrm{kg} \cdot \mathrm{K}), \rho_{3}=7700 \mathrm{~kg} / \mathrm{m}^{3}, \lambda_{3}=54 \mathrm{~W} /(\mathrm{m} \cdot \mathrm{K}) ; Q_{\mathrm{c}}=311.7 \cdot 10^{3} \mathrm{~J} / \mathrm{kg}$, where $C$ is heat capacity $(\mathrm{J} / \mathrm{kg} \cdot \mathrm{K}) ; \rho$ is density, $\mathrm{kg} / \mathrm{m}^{3} ; \lambda$ is thermal conductivity, $\mathrm{W} /(\mathrm{m} \cdot \mathrm{K})$; $Q_{\mathrm{c}}$ is the thermal effect of condensing agents, $\mathrm{J} / \mathrm{kg}$; codes " 1 ", "2" and "3" correspond to steams, condensate of working substance and tub's sides, respectively. 


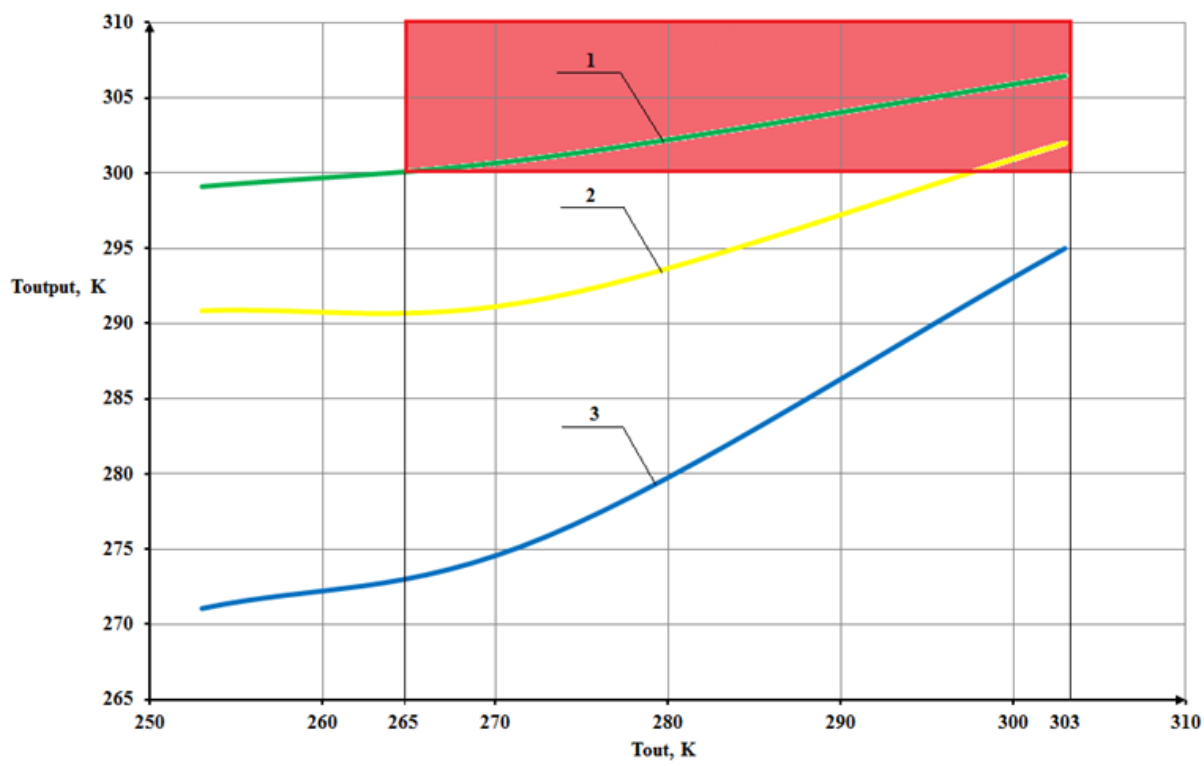

Fig. 3. Dependence of the output workflow $T_{\text {output }}$ temperature on the ambient air temperature: $1-T_{\text {input }}=449 \mathrm{~K} ; 2-T_{\text {input }}=426 \mathrm{~K} ; 3-T_{\text {input }}=405 \mathrm{~K}$.

Dependence analysis shows that the change in the output electric power of the considered mini-CHP Plant has a significant impact on the process of condensation in the tube and workflow $\mathrm{T}_{\text {output }}$ outlet temperature. It directly affects the efficient operation of a separate process and the work of the power plant as a whole. This result is explained by the fact that the speed of two-phase workflow through the condenser unit grows when the power of the power plant increases. It leads to an increase of the initial temperature of the working substance that affects the condensation process in a condenser, and hence the output temperature of the workflow from the condenser. In cases where the workflow appears in high ambient temperatures ( $265 \mathrm{~K}$ and above) there is a significant increase in the temperature of the working substance. Workflow temperature in the range between 300 $\mathrm{K}$ and above, is critical for the operation of power plants and may cause unrestricted operational states of mini-CHP plant.

\section{Conclusion}

Obtained results leads to the following conclusions:

1. It is established that the main parameter that significantly affects the output temperature of the working stream from the condenser $T_{\text {output }}$ is the ambient temperature and electric power, as well as the initial temperature of working medium at the condenser intake $\mathrm{T}_{\text {input }}$.

2. At high temperatures of outside air the reliability of autonomous energy unit work is reduced due the low efficiency of the method of additional heat transfer process trough the use of the fan for cooling the external surfaces of the condenser tubes. Significantly greater cooling efficiency can be achieved by using water cooling system of surfaces of the tubes of the condenser.

3. A high probability of exceeding the temperature of working medium at the zone of lubrication of slider bearings due to the wide range of ambient temperatures at different 
capacity of energy unit. According to the manufacturer the temperature of the working medium at the inlet to a zone of lubrication of the sliding bearings should not exceed 300 $\mathrm{K}$.

The obtained results allow to determine necessary and sufficiency conditions for accident free operation of the sliding bearings of the mini-CHP plant turbogenerator:

1. In case of energy units operation with nominal parameters (3000 W and $4000 \mathrm{~W})$ increases the probability of unexpected occurrences associated with the units shutdown in the ranges of the outside temperatures from $283 \mathrm{~K}$ and higher and $253 \mathrm{~K}$ and higher, respectively.

2. Increasing the area of heat transfer between the working medium and outside air allow to achieve the optimum temperature of the working stream at the outlet of the condenser, which will provide the decrease of the number of technical accidents associated with "mechanical failure of turbogenerator".

\section{Acknowledgments}

This work was financially supported by the Ministry of Education and Science of the Russian Federation (the scholarship of the President of the Russian Federation for young scientists and graduate students SP-1350.2015.1). The work at the field of modeling of heat and mass transfer processes near boundary between two layers was supported by the scientific schools grant NSH-7538-2016.8.

\section{References}

1. O.V. Vysokomornaya, V.S. Vysokomorny, P.A. Strizhak, Energy security and energy efficiency 1, 25 (2014)

2. P.I. Kononenko, V.G. Mikhailuts, A.J. Bezzutsev-Kondakov, Energetik 3, 43 (2007)

3. A.P. Livinskij, I.J. Red'ko, V.M. Filin, Energetik 4, 22 (2010)

4. Energy Strategy of Russia for the period until 2020 (Approved by the RF Government, 2003)

5. Eps operation \& maintenance manual (powered by CCVT) (ORMAT SYSTEMS LTD, 2006)

6. M.V. Piskunov, I.S. Voytkov, O.V. Vysokomornaya, V.S. Vysokomorny, EPJ Web Conf. 82 (2015) 